\title{
Using basic fibroblast growth factor nanoliposome combined with ultrasound-introduced technology to early intervene the diabetic cardiomyopathy
}

This article was published in the following Dove Press journal:

International Journal of Nanomedicine

18 February 2016

Number of times this article has been viewed

\author{
Ying-Zheng Zhao',2 \\ Ming Zhang' \\ Xin-Qiao Tian ${ }^{3}$ \\ Lei Zheng ${ }^{4}$ \\ Cui-Tao Lu', ${ }^{1,2}$
}

'College of Pharmaceutical Sciences,

Wenzhou Medical University,

${ }^{2}$ Department of Ultrasonography, The

Second Affiliated Hospital of Wenzhou

Medical University, Wenzhou,

Zhejiang, ${ }^{3}$ Department of Ultrasonic

Diagnosis, Henan Provincial People's

Hospital, Zhengzhou City, Henan,

${ }^{4}$ Department of Ultrasonography, The

First Affiliated Hospital of Wenzhou

Medical University, Wenzhou, Zhejiang,

People's Republic of China
Correspondence: Cui-Tao Lu

Department of Ultrasonography, The Second Affiliated Hospital of Wenzhou Medical University, Wenzhou City, Zhejiang Province 325000, People's

Republic of China

Email Ictuua@sina.com
Abstract: Basic fibroblast growth factor (bFGF)-loaded liposome (bFGF-lip) combined with ultrasound-targeted microbubble destruction (UTMD) technique was investigated to prevent diabetic cardiomyopathy (DCM). Cardiac function and myocardial ultrastructure were assessed. Terminal deoxynucleotidyl transferase (TdT)-mediated dUTP nick-end labeling (TUNEL) staining, immunohistochemistry staining, and Western blot assay were used to investigate the signal pathway underlying the expression of bFGF in DCM treatment. From Mason staining and TUNEL staining, bFGF-lip + UTMD group showed significant differences from the diabetes group and other groups treated with bFGF or bFGF-lip. The diabetes group showed similar results (myocardial capillary density, collagen volume fraction, and cardiac myocyte apoptosis index) to other bFGF treatment groups. Indexes from transthoracic echocardiography and hemodynamic evaluation also proved the same conclusion. These results confirmed that the abnormalities including diastolic dysfunctions, myocardial fibrosis, and metabolic disturbances could be suppressed by the different extents of twice-weekly bFGF treatments for 12 consecutive weeks (free bFGF or bFGF-lip +/- UTMD), with the strongest improvements observed in the bFGF-lip + UTMD group. The group combining bFGF-lip with UTMD demonstrated the highest level of bFGF expression among all the groups. The bFGF activated the PI3K/AKT signal pathway, causing the reduction of myocardial cell apoptosis and increase of microvascular density. This strategy using bFGF-lip and UTMD is a potential strategy in early intervention of DCM in diabetes.

Keywords: diabetic cardiomyopathy, prevention, bFGF-loaded liposome, targeted delivery

\section{Introduction}

Diabetes mellitus (DM) affects more than 180 million people around the world. Diabetic cardiomyopathy (DCM), caused by diabetes, is described as the structural and functional changes in the myocardium. ${ }^{1,2}$ The structural changes included fibrosis, apoptosis, and angiopathy of myocytes, and the functional changes included endothelium-myocytes uncoupling, impairment of contractility of cardiomyocytes, decrease in survival and differentiation of cardiac stem cells, and diastolic and systolic dysfunction. ${ }^{3-5}$ Nonetheless, the pathophysiology of DCM remains uncertain and novel therapeutic strategies for its prevention and rescue are urgently needed.

As a biological factor with high activity, basic fibroblast growth factor (bFGF) can induce endothelial and smooth muscle cell proliferation and angiogenesis in vivo, including the migration and proliferation of endothelial cells, vascular tube formation, and linkage to preexisting vascular networks. ${ }^{6,7}$ In addition, bFGF has shown to be an important survival (antiapoptotic) factor in a variety of cell types. ${ }^{8,9}$ Therefore, the administration of bFGF can reduce apoptosis of cardiomyocytes, improve reparative 
neovascularization, reduce deposition of interstitial fibrosis, and increase cardiac perfusion.

However, there is an urgent need to optimize the mode of bFGF delivery, aiming at inducing minimal impact on systemic tissues and retaining the bioactivity bFGF. Liposome is a vesicular structure consisting of hydrated lipid bilayer and is a nontoxic, biodegradable, and protective agent in depot preparations. Using liposome as the carrier of proteins can significantly improve the bioavailability of the proteins by protecting proteins from environmental conditions and acting for the long term by sustained release of active dug at the site. Therefore, liposome can be used as the suitable carrier for the delivery of bFGF in vivo. However, bFGF-encapsulated liposome (bFGF-lip) cannot significantly penetrate into myocardial tissue due to the lack of effective technology for targeted release and uptake in heart.

Recently, low-intensity ultrasound in combination with microbubble has acquired much attention as a safe method to trigger drug release. ${ }^{10,11}$ Numerous animal models and tissues have shown that the destruction of circulating contrast agent microbubbles with local low-frequency ultrasound can obviously enhance the release of drug from the liposome in the ultrasonic sites. ${ }^{12}$

In addition, ultrasound exposure alone had been proven to enhance the permeability of cell membrane, ${ }^{13}$ and addition of microbubble has a significant potentiating effect. ${ }^{14}$ Therefore, ultrasound-targeted microbubble destruction (UTMD) technique, conventionally used for clinical diagnosis, has a considerable promise as a tool for targeting release of drug from vectors.

In our previous study, the progress of cardiac functions after DCM can be effectively inhibited and even reversed by bFGF nanoparticles combined with UTMD technique. ${ }^{15}$ It gives a promising strategy for DCM-targeted therapy.

For modern medicine, early prevention is better than the cure after disease. In this study, we further investigated whether bFGF-lip combined with UTMD technique could be used in early prevention of DCM in rats with type 1 diabetes induced by streptozotocin (STZ). This study provides additional information for understanding of DCM recovery mechanism as well as developing new clinical treatment strategies.

To achieve an in-depth understanding of the therapeutic impact of the bFGF-lip/UTMD technique, a broad range of commonly used pathophysiological indicators of the heart conditions were measured in a DCM rat model induced by STZ. These measurements allowed thorough preclinical evaluation of the in vivo effects of 12 weeks bFGF-nanoparticle + UTMD treatment on the cardiac functions and related structural damages. Overall, this study has generated comprehensive data that are critical for the translation of this promising combination therapy of DCM, a frequently occurring and deadly disease.

\section{Materials and methods}

All reagents and animals used in this study were commercially available. The animals were handled according to the protocols approved by the ethical committee of Wenzhou Medical University and all the experiments were performed according to the National Institutes of Health Guide for the Care and Use of Laboratory Animals.

\section{Fabrication of phospholipid-based microbubbles}

Phospholipid-based microbubbles (PMBs) were prepared by the sonication-lyophilization method (Supplementary materials).

\section{Preparation and characterization of bFGF-lip}

The details of bFGF-lip preparation and characterization can be found in the Supplementary materials.

\section{Type I DM animal model}

STZ (Sigma-Aldrich, St Louis, MO, USA) was prepared as $1 \% \mathrm{STZ}$ solution in $0.1 \mathrm{M}$ citrate buffer ( $\mathrm{pH} 4.0-4.5)$. On days 3, 7, and 14 after STZ administration, the fasting blood glucose was measured from the tail vein using an autoanalyzer (Surestep, Roche, Germany). The animals with a fasting blood glucose exceeding $16.7 \mathrm{mM}$ after STZ treatments were used as the study group. Age- and sex-matched control animals were injected with citrate buffer. All animal experiments were performed after the approval of the protocol by the Institutional Animal Care and Use Committee in Wenzhou Medical University.

\section{Experimental design}

The experimental design and grouping are summarized in Table S1. After DM was induced, the study animals were randomly divided into seven groups: 1) DM group, 2) control group, 3) bFGF group, 4) bFGF-lip group, 5) UTMD group, 6) bFGF + UTMD group, and 7) bFGF-lip + UTMD group. For all animals, bFGF and PMBs were administered via tail vein injection twice weekly for 12 consecutive weeks (Supplementary materials). 


\section{UTMD treatment}

The experimental animals were anesthetized with intraperitoneal injection of $30 \mathrm{mg} / \mathrm{kg}$ sodium pentobarbital. They were placed in the supine position and the chests shaved. A 20-gauge cannula was inserted into the tail vein for infusion. A linear array transducer (15LSw.S probe, $12-14 \mathrm{MHz}$, Acuson Sequoia 512C system; Siemens Medical Solutions, Mountain View, CA, USA) was used to generate the UTMD effect. In the groups treated with UTMD, the linear array transducer was placed over the heart (short-axis view; depth $=3.0-4.0 \mathrm{~cm}$ ) to view papillary muscles of the left ventricle. There was no enhanced ultrasonic signals before the filling of microbubbles and bFGF-containing treatment. When a large number of PMBs were seen filling the heart, the microbubble destruction (MBD) function key (the key for controlling the bursting of microbubbles) attached to the machine was employed to disrupt PMB for UTMD (mechanical index $[\mathrm{MI}]=1.9$, exposure time $=10$ seconds, repeat three times with off intervals of 1 second to allow refill of the tissue with more microbubbles). After bursting, the microbubbles completely disappeared in heart. The UTMD treatment was performed by twice-weekly treatments (not each day) for 12 consecutive weeks and the total number of treatments was 24 .

\section{Measurement of cardiac dimensions and function \\ Transthoracic echocardiography}

M-mode images were obtained at the papillary muscle level from two-dimensional (2D) short-axis views. The indexes included for measurement were: left ventricular end-diastolic diameter (LVIDd), left ventricular posterior wall (LVPW), and left ventricular fraction shortening (LVFS).

The 2D images of three consecutive cardiac beats were also obtained with the highest possible frame rate from the same short-axis views at the papillary muscle level. Imaging depth was adjusted to $35-40 \mathrm{~mm}$, resulting in a temporal resolution at $70-90 \mathrm{~Hz}$.

All images were stored as standard Digital Imaging and Communication in Medicine format on magneto-optical disks and analyzed offline using Siemens Sygno US Workplace 3.01 software (Siemens Medical Solutions). In this study, the LV wall at midlevel from the short-axis view was divided into six segments according to the standard 16-segment model. ${ }^{16}$ The segments of the LV wall were plotted, the endocardial and epicardial borders were manually identified in one frame, and the borders in all other frames were automatically generated, allowing the operator to alter any of those contours. Next, segmental systolic radial peak velocity (Vs), circumferential strain (Sc), and strain rate (SRc) were obtained from velocity, strain, and strain rate curves, respectively.

\section{Hemodynamic measurements}

Animals were anesthetized with intraperitoneal injection of $30 \mathrm{mg} / \mathrm{kg}$ sodium pentobarbital. The trachea was exposed and the animal ventilator (HX-300; TME Technology Co., Ltd, Chengdu, People's Republic of China) was connected. The pericardial cavity was opened and a cardiac catheter (SPR869; Millar Instruments, Houston, TX, USA) was inserted into the LV from the apex. The LV systolic pressure (LVSP), end-diastolic pressure (LVEDP), and the maximum positive and negative $\mathrm{dp} / \mathrm{dt}\left(+\mathrm{dp} / \mathrm{dt}_{\max }\right.$ and $\left.-\mathrm{dp}_{\mathrm{dt}} \mathrm{dt}_{\max }\right)$ were measured by using an analog-to-digital converter. ${ }^{17}$

\section{Histological and molecular analyses}

After all the experiments were completed and the animals euthanized, the body weight (BW), heart weight (HW), and HW/BW ratio were measured. The HW/BW ratio was calculated according to the following formula:

$$
\text { HW/BW }=\frac{\text { Heart weight }(\mathrm{g})}{\text { Body weight }(\mathrm{g})} \times 100 \%
$$

LV tissue samples were obtained and stored in $2.5 \%$ glutaraldehyde for electron microscopic studies. Samples were also obtained and fixed in $10 \%$ formalin for paraffin section. The remaining tissues were stored in the freezer $\left(-80^{\circ} \mathrm{C}\right)$ for Western blot and other molecular analyses.

\section{Masson staining}

The collagen content of the left ventricle was detected by Masson staining. Briefly, $4 \mu \mathrm{m}$ of cardiac tissue sections were processed for staining with Masson's trichrome staining solution. Cardiac collagen volume fraction (CVF) was calculated by use of computer-assisted image analysis software (Image-Pro plus 6.0 imaging software, NIH).

\section{Myocardial capillary density}

Myocardial capillary density (MCD) was measured by counting the number of brown-stained capillaries in 20 visual fields using high power $(400 \times)$, and presented as the number of blood vessels per high-power field (n/hpf). CD31 immunohistochemical staining was used to identify capillaries to measure MCD. 


\section{Transmission electron microscopy}

Left ventricle samples were cut into $1 \mathrm{~mm}$ fragments and fixed in $2.5 \%$ glutaraldehyde for 4 hours for electron microscopic examination. The myocardial tissues were fixed in $1 \%$ osmic acid. After dehydration by acetone and embedded by epoxy resins, the myocardial tissue was sectioned at $1 \mu \mathrm{m}$ and stained with toluidine blue. Ultrathin sections were cut from the block and studied under a JEM-1230 (JEOL, Tokyo, Japan) transmission electron microscope.

\section{TUNEL staining}

The apoptotic cells of myocardium were evaluated by terminal deoxynucleotidyl transferase (TdT)-mediated dUTP nickend labeling (TUNEL) staining (Supplementary materials).

\section{Immunohistochemistry staining}

Cardiac capillary density (CD31) and antiapoptosis (phosphorylated AKT [pAKT])-related proteins were detected by immunohistochemistry staining (Supplementary materials).

\section{Western blot assay}

Expression of bFGF and apoptosis- and antiapoptosis-related proteins (AKT, pAKT, Bcl-2, Bax) was semiquantitatively detected by Western blot assay (Supplementary materials).

\section{Statistical analysis}

One-way analysis of variance (ANOVA), Student's $t$-test, or Kruskal-Wallis test was used for statistical comparison using the SAS 8.01 (1999-2000; SAS Institute Inc., Cary, NC, USA). The difference was considered to be statistically significant when the $P$-value was equal to or less than 0.05 .

\section{Results}

\section{Characterization of bFGF-lip}

Figure S1 and Table S2 showed the characteristics of the blank and bFGF-lip. The details about characterization of bFGF-lip can be found in the Supplementary materials.

\section{Improvement on heart-to-body weight ratio}

$\mathrm{BW}, \mathrm{HW} / \mathrm{BW}$ ratio, and serum glucose levels of the animals were obtained to evaluate the effect of UTMD treatment with bFGF-lip on their metabolism and cardiomyopathy. Figure S2 shows that animals in the six study groups had significantly lower BW and high serum glucose levels compared with the control animals but there was no significant difference in BW and serum glucose levels within the six study groups (Figure S2A and $\underline{\text { ) }}$. However, the HW/BW ratio of DM group was significantly higher than control group as well as the five groups with bFGF or bFGF-lip with or without UTMD treatment (Figure S2B).

\section{Prevention of left ventricle dysfunction in diabetic rats}

The results of the examination of LVIDd, LVPW, and LVFS by transthoracic echocardiography are given in Table S3. The results showed that the significant increase of LVPW $(P<0.05)$ as well as the decrease of LVIDd and LVFS $(P<0.05)$ was detected in DM animals at 3 months compared with normal animals, which indicated that the cardiac function of DM animals was significantly impaired under diabetic conditions. However, the significant increase of LVIDd and LVFS $(P<0.05)$ as well as the decrease of LVPW $(P<0.05)$ was detected in the groups after bFGF treatment. Moreover, the group receiving bFGF-lip combined with UTMD treatment showed highest LVIDd and LVFS as well as lowest LVPW among all the groups treated with bFGF, which indicated the bFGF-lip + UTMD had the best effect on the prevention of cardiac dysfunctions in diabetic rats by reversing all the indexes close to a normal level.

Myocardial velocity, strain, and strain rate data by velocity vector imaging (VVI) are shown in Table S4. Before any treatment, there was no statistically significant difference among control and all the study groups in Vs, Sc, and SRc values. After STZ administration and 12 weeks of treatment, there was a significant increase in Vs, Sc, and SRc (absolute values) in the bFGF treatment groups (groups 3, 4, 6, and 7) compared with the DM group. In addition, Vs, Sc, and SRc (absolute values) significantly increased in the group treated with bFGF-lip combined with UTMD (group 7) than other bFGF treatment groups without liposome or UTMD, and no difference in Vs, Sc, and SRc (absolute values) between group 7 and the control group was observed.

The invasive hemodynamic data by intracardiac catheters are shown in Table S5. Compared with control animals, the DM group showed significantly lower LVSP, $\mathrm{LV}+\mathrm{dp} / \mathrm{dt}_{\max }$, and $\mathrm{LV}-\mathrm{dp} / \mathrm{dt}_{\text {max }}$ but a higher LVEDP value $(P<0.05)$. In contrast to the DM group, the animals given bFGF treatment (groups $3,4,6$, and 7) exhibited significant improvement in LVSP, LVEDP, $\mathrm{LV}+\mathrm{dp} / \mathrm{dt}_{\max }$, and $\mathrm{LV}-\mathrm{dp} / \mathrm{dt}_{\max }$ values $(P<0.05)$. Moreover, the LVSP, LV $+\mathrm{dp} / \mathrm{dt}_{\text {max }}$, and LV - dp/dt ${ }_{\text {max }}$ values in the animals treated with bFGF-lip + UTMD were significantly higher and LVEDP values lower than the other bFGF treatment groups without liposome or UTMD, and no difference in LVSP, LVEDP, LV + dp/dt ${ }_{\text {max }}$, and LV $-\mathrm{dp} / \mathrm{dt}_{\text {max }}$ values were observed between group 7 and the control group. 


\section{Effects of UTMD treatment with bFGF-lip on myocardial fibrosis}

As shown in Figure 1, CVF significantly increased in the DM group, with altered and disorganized collagen network structure in the interstitial and perivascular areas, compared with control and other animals. In comparison with the DM group, CVF was significantly reduced in the groups treated with bFGF or bFGF-lip. Furthermore, CVF was significantly lower in the group treated with bFGF-lip combined with UTMD than other groups without liposome or UTMD, and there was no difference in CVF between this group and the control group.

\section{Effects of UTMD treatment with bFGF-lip on electron microscopic findings}

As shown in Figure 2, alterations in myofilaments, Z-line myofibers, and degeneration and destruction in myofibrils were observed in the myocardial samples by electron microscopy in the study animals compared with the control ones. In addition, the mitochondria of cardiomyocytes in the DM group showed loss of cristae and granular matrix with vacuolization (Figure 2A). There were similar alterations in the ultrastructures of the cardiomyocytes, as observed by electron microscope, in the group with UTMD treatment alone compared with the DM group (Figure 2E). However, few alterations in the myocardial ultrastructures were observed in the bFGF treatment groups (Figure 2C, D, F, and G). Furthermore, there were well-organized and symmetric myofibrils in the Z-lines and sarcomeres as well as relatively well-integrated ultrastructures in the mitochondria (Figure 2G) in the group treated with bFGFlip combined with UTMD, compared with other treatment and DM groups.

\section{A}

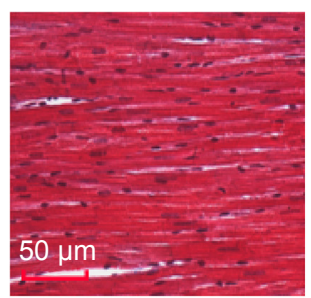

Control

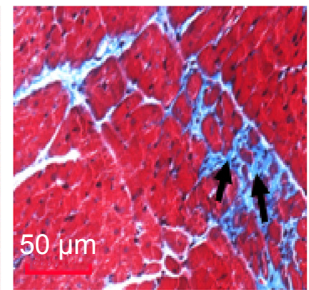

DM

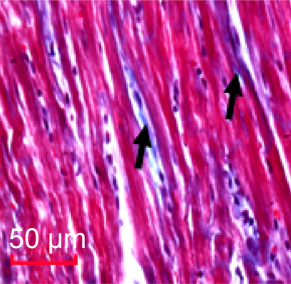

bFGF

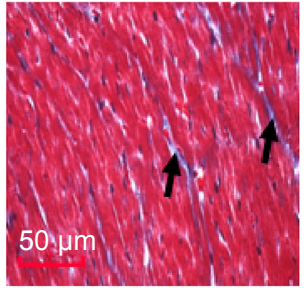

bFGF-lip

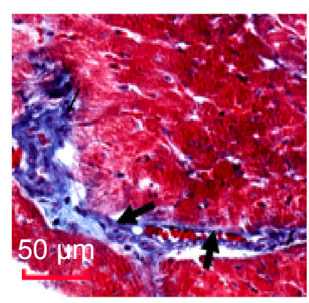

UTMD

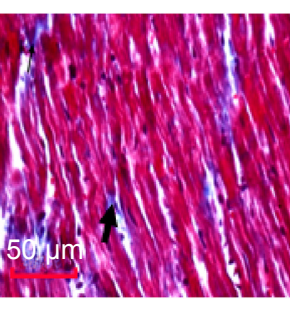

bFGF + UTMD

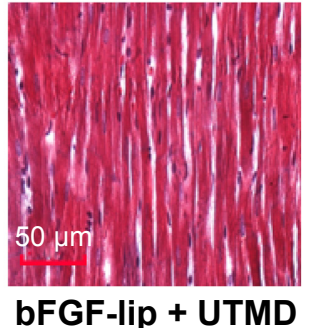

B

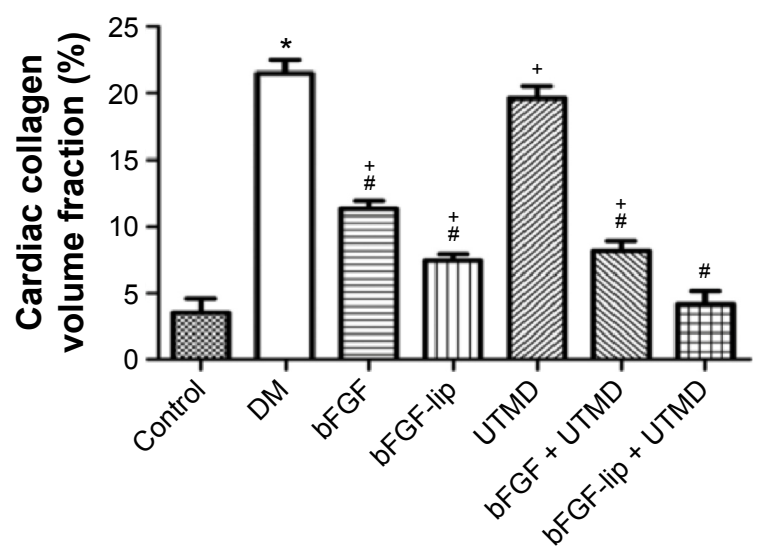

Figure I DM-induced cardiomyocyte interstitial fibrosis under bFGF-lip treatment.

Notes: (A) Representative pictures of myocardial tissue sections stained with Masson trichrome (400 $\times)$. N=6 per group. Arrow indicates myocardial fibrosis stained in blue. (B) Quantitative analysis of cardiac collagen volume fraction. $\mathrm{N}=8$ per group. Data are expressed as mean $\pm \mathrm{SD}$. ${ }^{* P}<0.01$ vs control group; ${ }^{*} P<0.05$ vs $\mathrm{DM}$ group; ${ }^{+P}<0.05$ vs bFGF-lip + UTMD group.

Abbreviations: DM, diabetes mellitus; SD, standard deviation; bFGF, basic fibroblast growth factor; bFGF-lip, bFGF-loaded liposome; UTMD, ultrasound-targeted microbubble destruction. 


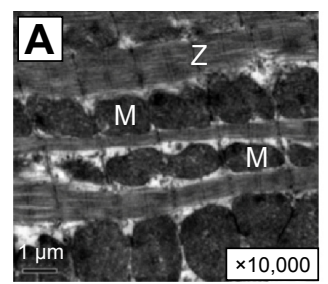

Control

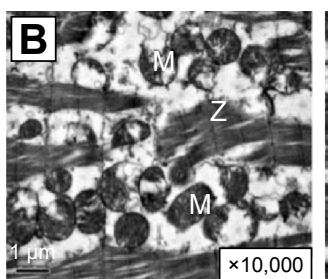

DM

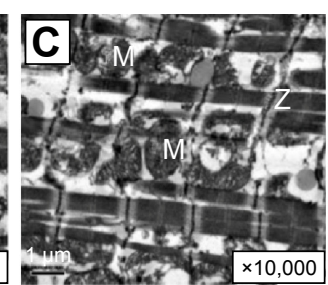

bFGF

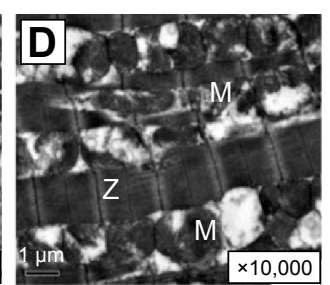

bFGF-lip

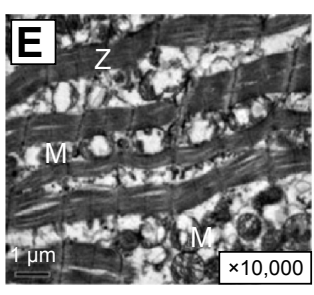

UTMD

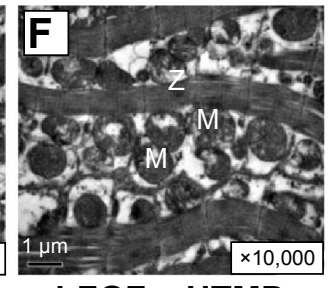

bFGF + UTMD

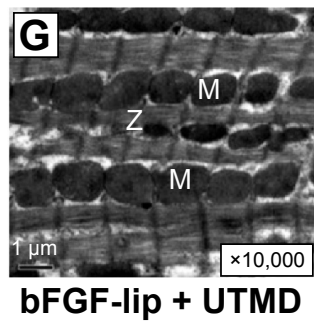

Figure 2 Preventing cardiomyocyte from DM injury by bFGF-lip.

Notes: Electron micrographs (magnification $\times 10,000$ ) of left ventricular heart muscle sections from the rats of each group. $\mathrm{N}=8 \mathrm{per}$ group. The $Z$ represents the $Z$-line in myofiber and the $M$ represents the mitochondria. As shown in (A-G), alterations in myofilaments and the Z-lines of myofiber, destruction and loss of myofibrils over sarcomere units, and swollen mitochondria were observed in DM group rats. The damage to myocardial cell in bFGF-treated rats was obviously attenuated particularly in the group that combined bFGF-lip with UTMD compared with DM group and other groups.

Abbreviations: DM, diabetes mellitus; bFGF, basic fibroblast growth factor; bFGF-lip, bFGF-loaded liposome; UTMD, ultrasound-targeted microbubble destruction.

\section{Effects of UTMD treatment with bFGF- lip on expression of caspase-3, Bcl-2, Bax, and bFGF levels}

As shown in Figure 3, increased expression of caspase-3 and Bax but reduced expression of Bcl-2 was observed in the DM group compared with the control group $(P<0.05)$. Remarkably, the groups treated with bFGF had significantly lower expression level of caspase- 3 and Bax as well as higher expression level of Bcl-2 than the DM group. Quantitative analysis also confirmed that the group combining bFGF-lip with UTMD could significantly upregulate Bcl-2 (Figure 3E) and downregulate Bax (Figure 3E) and caspase-3 (Figure 3B) expression compared with other treatment groups $(P<0.05)$.

Furthermore, the bFGF levels in the hearts of bFGFtreated groups had significantly higher levels of bFGF than DM and control groups by Western blot (Figure 3C). Moreover, the group that received combined bFGF-lip and UTMD treatment showed higher level of bFGF levels than the other bFGF-treated groups, which was confirmed by quantitative analyses (Figure 3D).

\section{Effects of UTMD treatment with bFGF-lip on cardiomyocyte apoptosis}

As shown in Figure 4, the TUNEL-staining positive cells and the apoptotic indexes were significantly increased in the DM group compared with the control animals $(P<0.01)$. However, bFGF treatment groups significantly attenuated DM-induced myocyte apoptosis in the study groups $(P<0.01)$. Among the
bFGF-treated groups, the group treated with bFGF-lip combined with UTMD had a significantly lower apoptotic index than the other treatment groups. Quantitative analysis also demonstrated that the TUNEL-positive cardiomyocytes were significantly less in the group receiving combined bFGF-lip and UTMD than the other treatment groups $(P<0.05)$.

\section{Effects of UTMD treatment with bFGF-lip on expression of pAKT and pAKT/AKT}

As shown in Figure 5A and B, immunohistochemical staining confirmed that the expression of pAKT was significantly reduced in the DM group compared with the control group. There was a significantly higher expression of pAKT in bFGFtreated groups compared with DM group $(P<0.05)$. Moreover, the group receiving combined bFGF-lip and UTMD treatment had higher pAKT level than the other bFGF-treated groups $(P<0.05)$. Western blot analysis also confirmed that the ratio of $\mathrm{pAKT} / \mathrm{AKT}$ was significantly higher in the group treated with combined bFGF-lip and UTMD compared with other bFGF-treated groups $(P<0.05$, Figure $5 \mathrm{C}$ and $\mathrm{D})$.

\section{Effects of UTMD treatment with bFGF-lip on myocardial capillary density}

As shown in Figure 6A, MCD was significantly lower in the DM group compared to the control group $(P<0.01)$. The bFGF-treated groups had significantly higher MCD than the DM group $(P<0.05)$. Additionally, the group treated with bFGF-lip and UTMD had higher MCD than the other bFGF-treated groups $(P<0.05)$. 
A

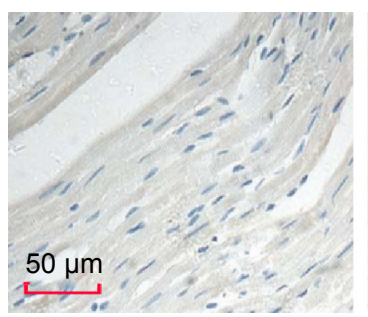

Control

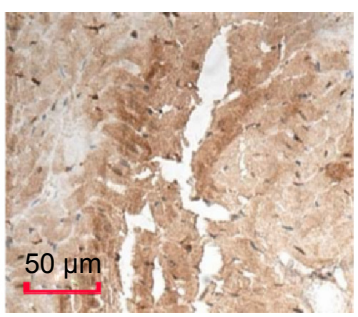

DM

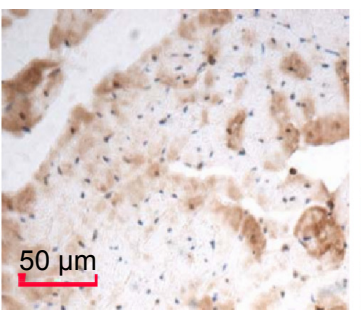

bFGF

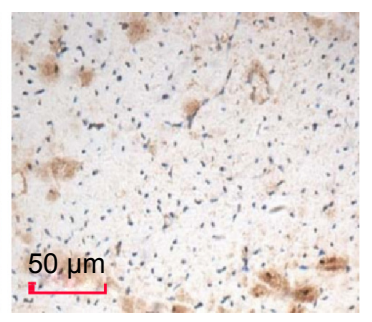

bFGF-lip

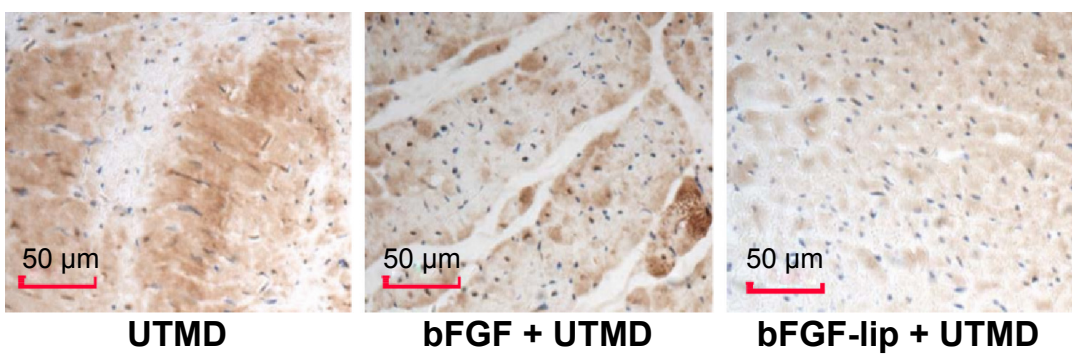

B
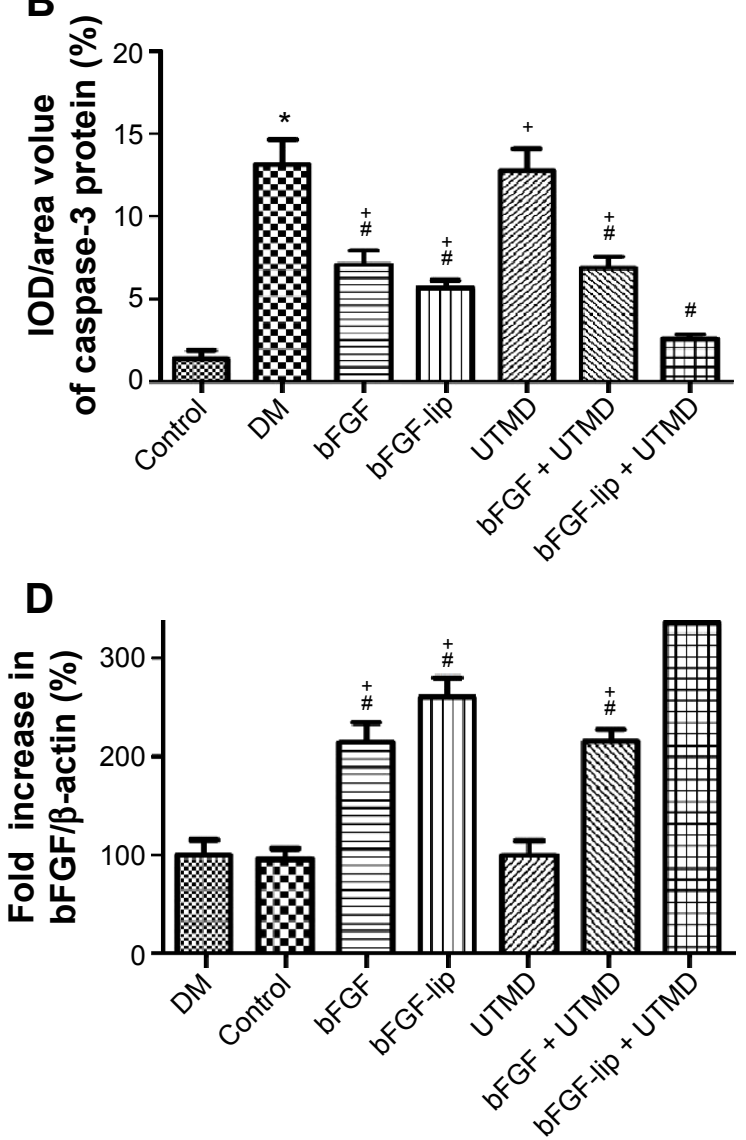

C

Figure 3 Expression of caspase-3, Bcl-2, Bax, and bFGF in diabetic hearts under bFGF-lip treatment.

Notes: (A) Representative immunohistochemical staining of caspase-3 expression, magnification =400x; (B) quantitative analyses of the expression of caspase-3; (C) Western blot analysis of bFGF, Bcl-2, and Bax expression; (D, E) quantitative analyses of the expression of bFGF, Bcl-2, and Bax, respectively; $n=8$ per group. Data are expressed as mean \pm SD. $* P<0.05$ vs control group; ${ }^{*} P<0.05$ vs $D M$ group; ${ }^{P}<<0.05$ vs bFGF-lip + UTMD group.

Abbreviations: DM, diabetes mellitus; SD, standard deviation; bFGF, basic fibroblast growth factor; bFGF-lip, bFGF-loaded liposome; UTMD, ultrasound-targeted microbubble destruction; IOD, integral optical density.

\section{Discussion}

Here, we report that using bFGF-loaded liposome with UTMD technique prevents the development of DCM in rats with STZ-induced type 1 diabetes. As one member of FGFs, bFGF can bind to tyrosine kinase receptors and mediate mitogenic activities, stimulating tissue growth, cellular proliferation, and migration; ${ }^{18,19}$ therefore, bFGF has a potential for prevention of DCM. However, it remains 


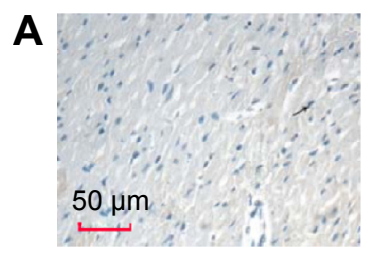

Control

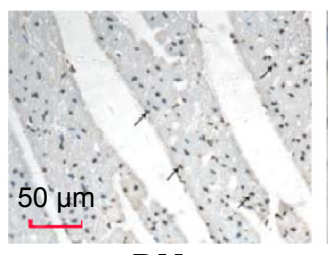

DM

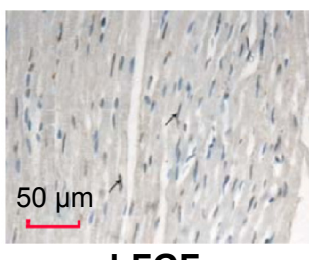

bFGF

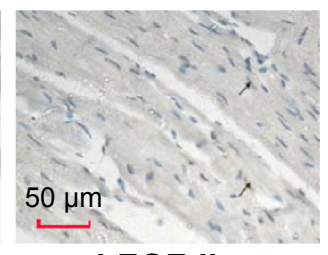

bFGF-lip
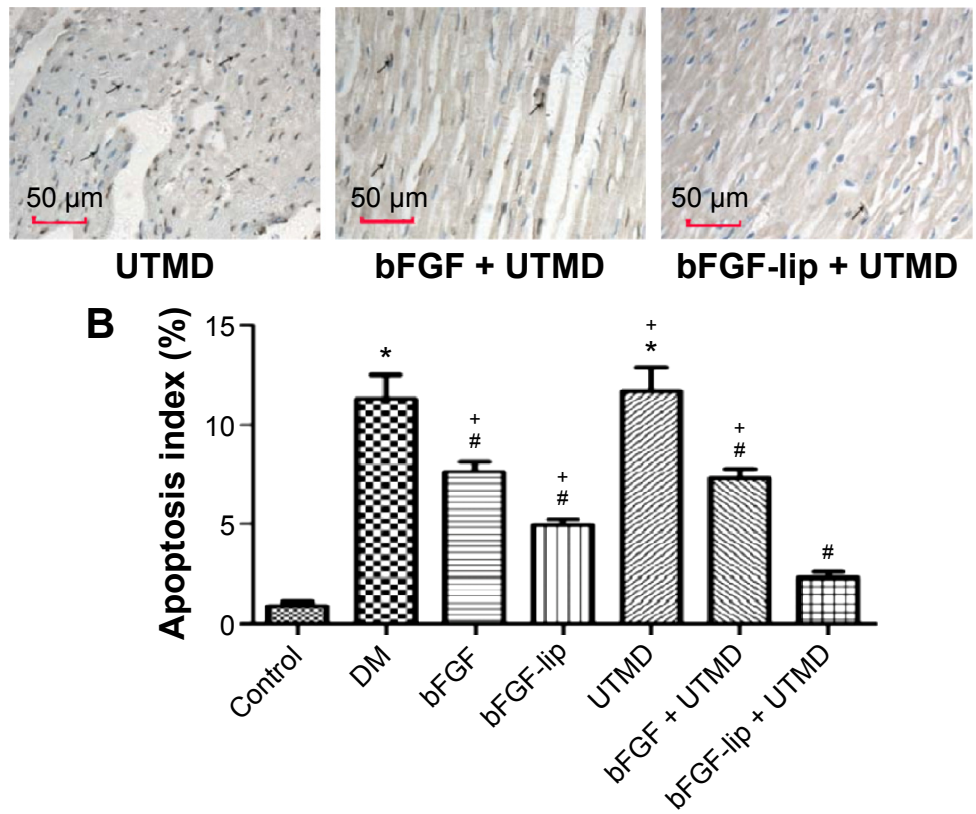

Figure 4 Prevention of DM-induced cardiomyocytes apoptosis under bFGF-lip treatment.

Notes: (A) Representative pictures of TUNEL assay (400x, arrow indicates TUNEL-positive cardiomyocytes stained in brown); (B) quantitative analysis of cardiomyocyte apoptosis index. $\mathrm{N}=8$ per group. Data are expressed as mean $\pm \mathrm{SD}$. ${ }^{*} P<0.01$ vs control group; ${ }^{*} P<0.01$ vs $D M$ group; ${ }^{+P}<0.05$ vs $b F G F-l i p+U T M D$ group.

Abbreviations: DM, diabetes mellitus; SD, standard deviation; bFGF, basic fibroblast growth factor; bFGF-lip, bFGF-loaded liposome; UTMD, ultrasound-targeted microbubble destruction; TUNEL, Terminal deoxynucleotidyl transferase (TdT)-mediated dUTP nick-end labeling.

difficult to apply bFGF in clinics due to the lack of an efficient delivery system.

It is evident that liposome is a nontoxic, biodegradable, and nonimmunogenic nonviral vector and can effectively deliver macromolecular proteins to their therapeutic targets. Moreover, UTMD technique is a promising method for targeting the delivery of bioactive agents to cardiac tissue and can also improve drug penetration into the myocardial cell. ${ }^{20}$ The images captured by the linear array transducer at different stages of the UTMD process are shown in Figure 7A-D. It can be seen that the microbubbles begin to accumulate in the ventricle of the heart (Figure 7B) and intensely burst during the MBD stage (Figure 7C). According to a previous report, sonoporation on cell membranes resulting from UTMD would continue for a period of time because the enlarged membrane pores needed time to restore back to normal size. ${ }^{21}$ Even though microbubbles completely disappeared in the heart (Figure 7D), the lasting sonoporation effect on myocardial cells remained beneficial for the absorption of residual bFGF into the blood circulation.
In our previous study, nonviral vector combined with UTMD technique has been proved as an effective strategy to deliver bFGF to reverse the progress of DCM. ${ }^{15}$ In this study, we further used the novel combined heart-targeted delivery strategy to explore the potential of bFGF on preventing diabetes-induced cardiac dysfunction and microvascular rarefaction. In order to maintain the bioactivity of bFGF, novel Poloxamer 188-grafted heparin copolymer and water-in-water emulsion technique were used in bFGF-lip preparation. From the characteristic evaluation, bFGF-lip was shown to have satisfied micrographs, high encapsulation efficiency, and good bioactivity in vitro. Western blot analysis of heart tissues at 12 weeks of diabetic development confirmed that the group combining bFGF-lip with UTMD had the highest level of bFGF among all the groups, which indicates that the liposome-loaded bFGF combined with UTMD can effectively deliver bFGF into myocardial tissue.

Throughout the period of development of diabetes, left ventricle diastolic dysfunction is the earliest abnormality 

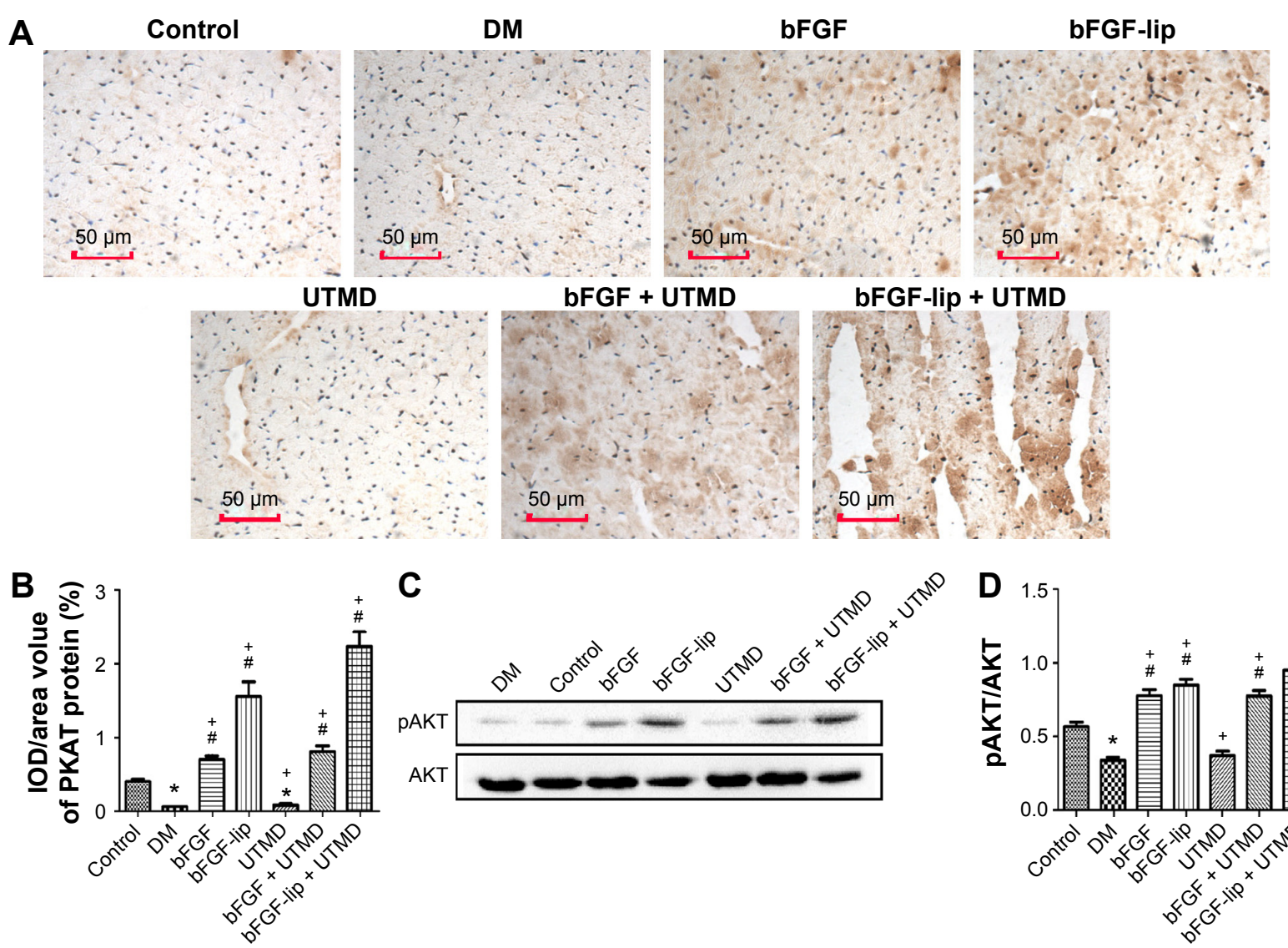

Figure 5 Enhanced expression of PAKT and upregulated ratio of pAKT/AKT in diabetic rats under bFGF-lip treatment.

Notes: (A) Representative immunohistochemical staining of pAKT expression (400x); (B) quantitative analyses of the expression of caspase-3; (C) Western blot analysis of the expression of $\mathrm{pAKT}$ and AKT; (D) quantitative analyses of the ratio of $\mathrm{pAKT} / \mathrm{AKT}$. $\mathrm{N}=8$ per group. Data are expressed as mean $\pm \mathrm{SD}$. $* \mathrm{P}<0.05$ vs control group; ${ }^{*}<0.05$ vs DM group; ${ }^{+} P<0.05$ vs bFGF-lip + UTMD group.

Abbreviations: pAKT, phosphorylated AKT; DM, diabetes mellitus; SD, standard deviation; bFGF, basic fibroblast growth factor; bFGF-lip, bFGF-loaded liposome; UTMD, ultrasound-targeted microbubble destruction; IOD, integral optical density.

observed in the heart of diabetic rats and systolic dysfunction supervenes at later stages of the DCM.22 Consistent with the previous reports, ${ }^{23}$ transthoracic echocardiography and hemodynamic evaluation showed that after 12 weeks the untreated diabetic rats in the present study were characterized by declined diastolic and systolic myocardial performance, which was associated with excess collagen accumulation and cardiac damage. Moreover, increased expression of proapoptotic proteins, caspase-3 and Bax, coupled with decreased expression of antiapoptotic protein, Bcl-2, and inactivation of prosurvival pathways of phosphatidylinositol 3-kinase (PI3K)/AKT finally resulted in cell apoptosis, cardiac fibrosis, and reduction of myocardial microvascular density in the untreated diabetic heart. In contrast, we have demonstrated that long-term treatment with bFGF-lip combined with UTMD can effectively prevent the development of such characteristic alterations of DCM. The mechanism by which bFGF can prevent the development of DCM in the diabetic rats can be explained as follows: first, bFGF activates the important AKT signaling pathway in the diabetic hearts, and thus reduces the apoptosis of myocardial cells and increases the function of diabetic heart. $\mathrm{AKT}$, as a serine/threonine kinase, is downstream of PI3K and influences every aspect of cellular functions including cell growth, survival, proliferation, and metabolism. As the activated form of AKT, phosphorylated AKT (pAKT) can reduce myocardial apoptosis in response to ischemia/reperfusion injury. ${ }^{24}$ Declining pAKT is also linked to increased apoptosis in pacing-induced heart failure. ${ }^{25}$ It is known that bFGF, as a stimulus, is capable of increasing AKT kinase activity by up to 40 -fold in a PI3K-dependent manner. ${ }^{26} \mathrm{After}$ the activation of AKT by bFGF, it can influence the activities of many downstream targets, such as inhibition of caspase-3 activity and exertion of antiapoptotic effects by phosphorylation and translocation of Bax to the mitochondria and reducing the release of chrome. ${ }^{27}$ Our results confirmed that the bFGF-lip combined with UTMD had the best potential to deliver bFGF into myocardial cells and reduce the apoptosis 


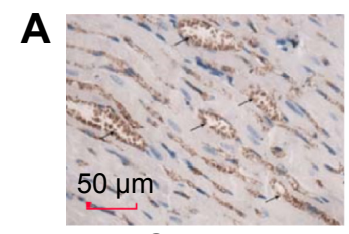

Control

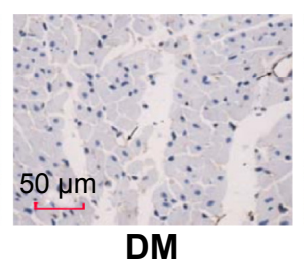

DM

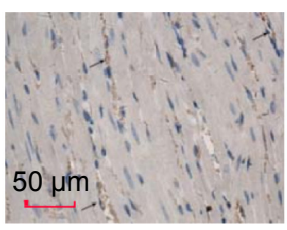

bFGF

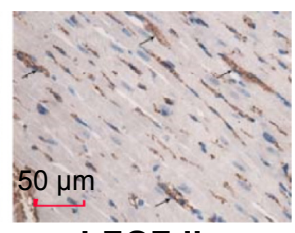

bFGF-lip
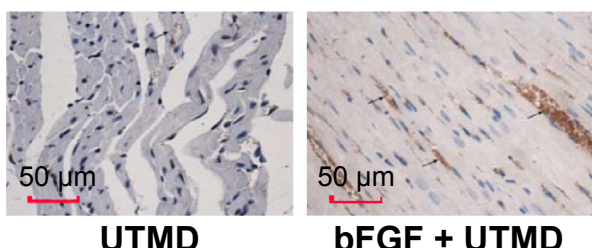

bFGF + UTMD

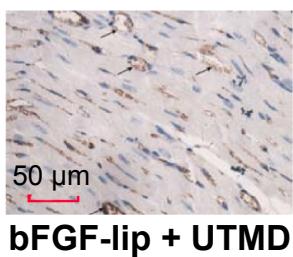

B

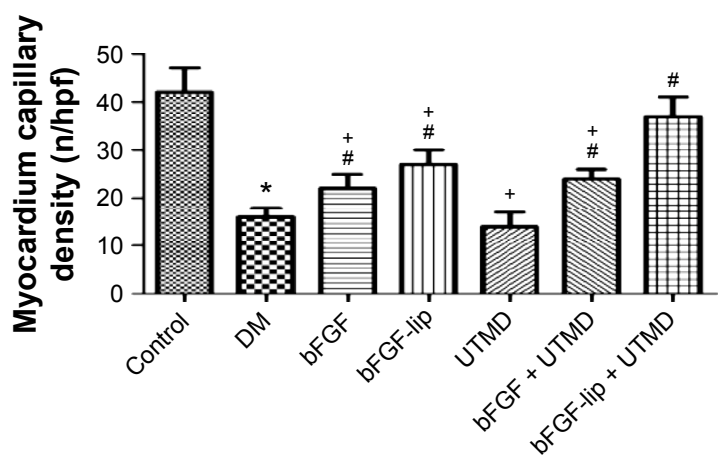

Figure 6 Myocardial microvascular density of the diabetic heart and myocardial perfusion under bFGF-lip treatment.

Notes: (A) Representative immunohistochemical staining of CD3I expression. (200×, arrow indicates CD3I-positive cardiomyocytes stained in brown); (B) quantitative analyses of the value of MCD. $N=8$ per group. Data are expressed as mean $\pm S D$. $* P<0.05$ vs control group; ${ }^{\#}<<0.05$ vs $D M$ group; ${ }^{+} P<0.05$ vs bFGF-lip $+U T M D$ group. Abbreviations: DM, diabetes mellitus; SD, standard deviation; MCD, myocardial capillary density; bFGF, basic fibroblast growth factor; bFGF-lip, bFGF-loaded liposome; UTMD, ultrasound-targeted microbubble destruction; $\mathrm{n} / \mathrm{hpf}$, number of blood vessels per high-power field.
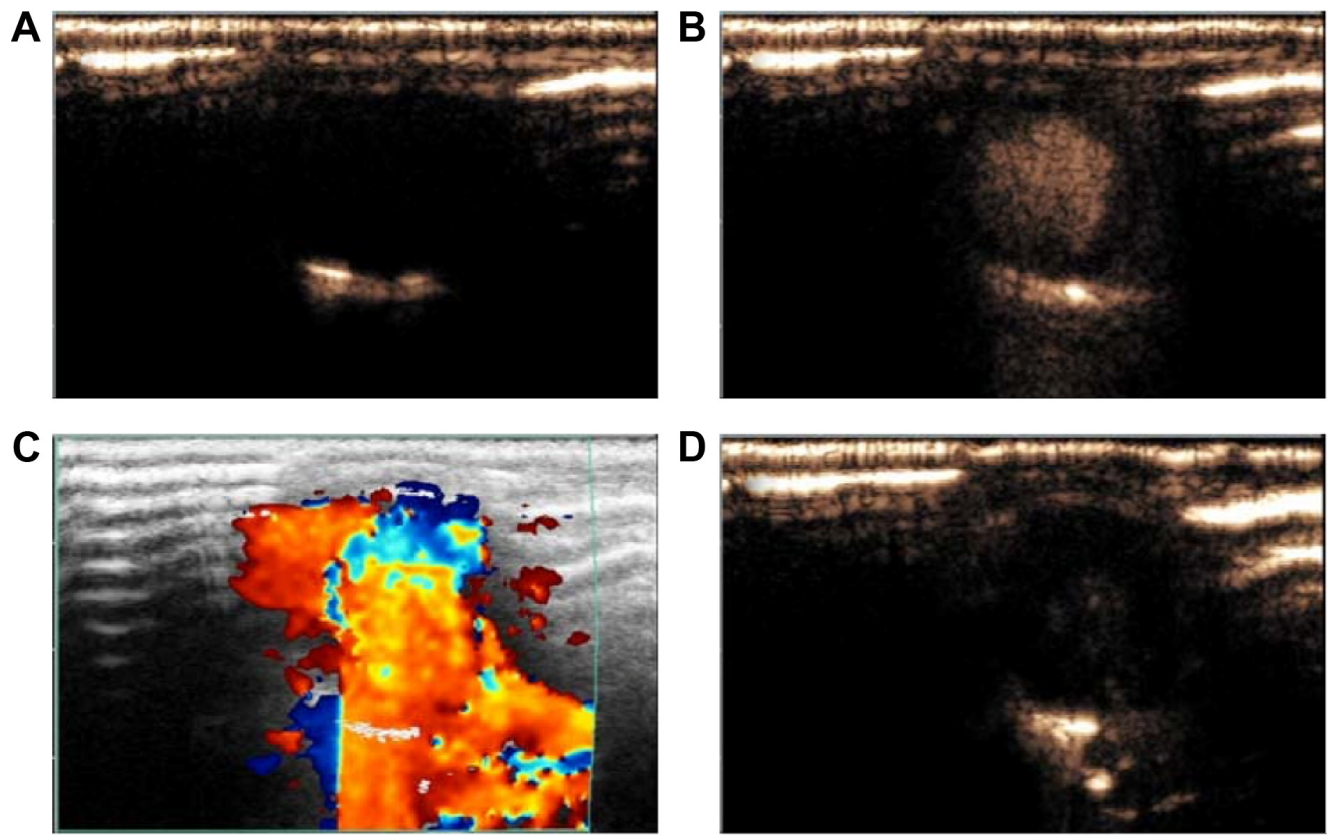

Figure 7 Images captured by the linear array transducer at different stages of the UTMD process.

Notes: (A) Ultrasound images before the filling of microbubble agent, following injection; (B) left ventricular myocardium was gradually filled with microbubble agent; (C) targeted bursting of microbubbles by MBD function; (D) ultrasound images after UTMD treatment.

Abbreviations: UTMD, ultrasound-targeted microbubble destruction; MBD, microbubble destruction. 
and fibrosis of cardiomyocytes via activating the AKT signal pathway. We also found that the administration of bFGF had little effect on the blood glucose levels and BW of diabetic rats. Second, bFGF can improve cardiac perfusion by increasing microvascular density. "Microangiopathy" has been demonstrated in the myocardium of diabetic rats and is one of the marked characteristics of DCM. ${ }^{28}$ As the diabetes progresses, "microangiopathy" exerts changes in the morphology and density. The changes in the morphology of microvasculature include thickening of the capillary basement membrane, medial thickening of the arterial wall, and perivascular fibrosis. ${ }^{29,30}$ Otherwise, the density of the microvessel in the diabetics is significantly decreased due to the apoptosis of endothelial cells compared to normal myocardium, so that it reduces the perfusion of the diabetic heart. However, bFGF can activate the PI3K/AKT signaling pathway according to the mechanism described earlier, and can thus reduce the apoptosis of endothelial cells, increase the density of the microvessels, and reduce pervascular fibrosis. In a previous study, Yamamoto et al showed that the injected bFGF into transmyocardial channels in a pig model of chronic myocardial ischemia can significantly increase vascular density by almost $40 \%$ compared with the control group..$^{31}$ In our study, the CD31 immunohistochemical staining also confirmed that the bFGF-lip combined with UTMD can effectively deliver bFGF into the hearts of diabetic rats and exert the effect of bFGF on the increased microvascular density.

\section{Conclusion}

In conclusion, our study demonstrated that bFGF-lip combined with UTMD showed great potential in preventing DCM development. Under further study in clinical application, this combined strategy might be used as an effective option for early intervention of DCM in diabetic patients.

\section{Acknowledgments}

This research was supported by National Natural Science Foundation of China (Grant Nos 81360195, 81301982, 81571392, and 81272160), Zhejiang Provincial Foundation for Health Department (Grant No 2015ZDA023), and grant from Wenzhou Bureau of Science and Technology (Grant No Y20140726).

\section{Disclosure}

The authors report no conflicts of interest in this work. The authors alone are responsible for the content and writing of this paper.

\section{References}

1. Goyal BR, Mehta AA. Diabetic cardiomyopathy: pathophysiological mechanisms and cardiac dysfuntion. Hum Exp Toxicol. 2013;32: 571-590.

2. Miki T, Yuda S, Kouzu H, Miura T. Diabetic cardiomyopathy: pathophysiology and clinical features. Heart Fail Rev. 2013;18: 149-166.

3. Delucchi F, Berni R, Frati C, et al. Resveratrol treatment reduces cardiac progenitor cell dysfunction and prevents morpho-functional ventricular remodeling in type-1 diabetic rats. PLoS One. 2012;7:e39836.

4. Fowlkes V, Clark J, Fix C, et al. Type II diabetes promotes a myofibroblast phenotype in cardiac fibroblasts. Life Sci. 2013;92: 669-676.

5. Zhang H, Zhang Y, Li Z, et al. Left ventricular radial systolic dysfunction in diabetic patients assessed by myocardial acceleration derived from velocity vector imaging. J Ultrasound Med. 2012;31:1179-1186.

6. Folkman J, Klagsbrun M. Angiogenic factors. Science. 1987;235: 442-447.

7. Montesano R, Vassalli JD, Baird A, Guillemin R, Orci L. Basic fibroblast growth factor induces angiogenesis in vitro. Proc Natl Acad Sci U S A. 1986;83:7297-7301.

8. Desire L, Courtois Y, Jeanny JC. Endogenous and exogenous fibroblast growth factor 2 support survival of chick retinal neurons by control of neuronal neuronal bcl-x(L) and bcl-2 expression through a fibroblast berowth factor receptor 1- and ERK-dependent pathway. J Neurochem. 2000;75:151-163.

9. Wang Y, He H, Zigler JS Jr, et al. bFGF suppresses serum-deprivationinduced apoptosis in a human lens epithelial cell line. Exp Cell Res. 1999;249:123-130.

10. Duvs.hani-Eshet M, Machluf M. Therapeutic ultrasound optimization for gene delivery: a key factor achieving nuclear DNA localization. J Control Release. 2005;108:513-528.

11. Kim HJ, Greenleaf JF, Kinnick RR, Bronk JT, Bolander ME. Ultrasoundmediated transfection of mammalian cells. Hum Gene Ther. 1996;7: 1339-1346.

12. Chappell JC, Song J, Burke CW, Klibanov AL, Price RJ. Targeted delivery of nanoparticles bearing fibroblast growth factor- 2 by ultrasonic microbubble destruction for therapeutic arteriogenesis. Small. 2008;4: 1769-1777.

13. Rapoport N, Kennedy AM, Shea JE, Scaife CL, Nam KH. Ultrasonic nanotherapy of pancreatic cancer: lessons from ultrasound imaging. Mol Pharm. 2010;7:22-31.

14. Tian JL, Zhao YZ, Jin Z, et al. Synthesis and characterization of Poloxamer 188-grafted heparin copolymer. Drug Dev Ind Pharm. 2010; 36:832-838

15. Zhao YZ, Tian XQ, Zhang M, et al. Functional and pathological improvements of the hearts in diabetes model by the combined therapy of bFGF-loaded nanoparticles with ultrasound-targeted microbubble destruction. J Control Release. 2014;186C:22-31.

16. Zhao YZ, Tian XQ, Zhang M, et al. Functional and pathological improvements of the hearts in diabetes model by the combined therapy of bFGF-loaded nanoparticles with ultrasound-targeted microbubble destruction. J Control Release. 2014;186:22-31.

17. Wang J, Song Y, Elsherif L, et al. Cardiac metallothionein induction plays the major role in the prevention of diabetic cardiomyopathy by zinc supplementation. Circulation. 2006;113:544-554.

18. Khurana R, Simons M. Insights from angiogenesis trials using fibroblast growth factor for advanced arteriosclerotic disease. Trends Cardiovasc Med. 2003;13:116-122.

19. Ornitz DM, Itoh N. Fibroblast growth factors. Genome Biol. 2001;2: REVIEWS3005.

20. Fujii $\mathrm{H}, \mathrm{Li} \mathrm{SH}, \mathrm{Wu}$ J, et al. Repeated and targeted transfer of angiogenic plasmids into the infarcted rat heart via ultrasound targeted microbubble destruction enhances cardiac repair. Eur Heart J. 2011;32:2075-2084.

21. Zhao YZ, Luo YK, Lu CT, et al. Phospholipids-based microbubbles sonoporation pore size and reseal of cell membrane cultured in vitro. J Drug Target. 2008;16:18-25. 
22. Nunes S, Soares E, Fernandes J, et al. Early cardiac changes in a rat model of prediabetes: brain natriuretic peptide overexpression seems to be the best marker. Cardiovasc Diabetol. 2013;12:44.

23. Meloni M, Descamps B, Caporali A, et al. Nerve growth factor gene therapy using adeno-associated viral vectors prevents cardiomyopathy in type 1 diabetic mice. Diabetes. 2012;61:229-240.

24. Armstrong SC. Protein kinase activation and myocardial ischemia/ reperfusion injury. Cardiovasc Res. 2004;61:427-436.

25. Ananthakrishnan R, Moe GW, Goldenthal MJ, Marin-Garcia J. Akt signaling pathway in pacing-induced heart failure. Mol Cell Biochem. 2005;268:103-110.

26. Datta SR, Brunet A, Greenberg ME. Cellular survival: a play in three Akts. Genes Dev. 1999;13:2905-2927.

27. Linseman DA, Butts BD, Precht TA, et al. Glycogen synthase kinase3 beta phosphorylates Bax and promotes its mitochondrial localization during neuronal apoptosis. J Neurosci. 2004;24:9993-10002.
28. Factor SM, Minase T, Sonnenblick EH. Clinical and morphological features of human hypertensive-diabetic cardiomyopathy. Am Heart J. 1980;99:446-458.

29. Kawaguchi M, Techigawara M, Ishihata T, et al. A comparison of ultrastructural changes on endomyocardial biopsy specimens obtained from patients with diabetes mellitus with and without hypertension. Heart Vessels. 1997;12:267-274.

30. Yarom R, Zirkin H, Stammler G, Rose AG. Human coronary microvessels in diabetes and ischaemia. Morphometric study of autopsy material. J Pathol. 1992;166:265-270.

31. Yamamoto N, Kohmoto T, Roethy W, et al. Histologic evidence that basic fibroblast growth factor enhances the angiogenic effects of transmyocardial laser revascularization. Basic Res Cardiol. 2000;95: 55-63.
International Journal of Nanomedicine

\section{Publish your work in this journal}

The International Journal of Nanomedicine is an international, peerreviewed journal focusing on the application of nanotechnology in diagnostics, therapeutics, and drug delivery systems throughout the biomedical field. This journal is indexed on PubMed Central, MedLine, CAS, SciSearch ${ }^{\circledR}$, Current Contents ${ }^{\circledR} /$ Clinical Medicine,

\section{Dovepress}

Journal Citation Reports/Science Edition, EMBase, Scopus and the Elsevier Bibliographic databases. The manuscript management system is completely online and includes a very quick and fair peer-review system, which is all easy to use. Visit http://www.dovepress.com/ testimonials.php to read real quotes from published authors. 